\title{
Solutions of a system of second-order ordinary differential equations
}

\author{
Kurt W. Tomantschger
}




\title{
SOLUTIONS OF A SYSTEM OF SECOND-ORDER ORDINARY DIFFERENTIAL EQUATIONS
}

\author{
Kurt W. TOMAnTsChGeR \\ Department of Mathematics, Technical University of Graz \\ Steyrergasse 30, A-8010 Graz, Austria \\ toman@weyl.math.tu-graz.ac.at
}

[Received March 24, 2000]

\begin{abstract}
A general solution of a coupled system of second-order ordinary differential equations is obtained. The solution is represented by modified Bessel functions of the first and second kind. The corresponding boundary conditions are also given. The boundary value problem describes the motion of a micropolar suspension between two coaxial cylinders, and the two unknown functions are, respectively, the velocity and the velocity of microrotation of the micropolar theory.
\end{abstract}

Mathematical Subject Classification: 34B05, 33C10

Keywords: Coupled linear ordinary differential equations, modified Bessel functions, MacDonald functions

\section{Introduction}

Classical continuum mechanics is not sufficient to describe the behaviour of certain materials (granular materials, fluid suspensions, liquid crystals, blood flow, polymeric substances, composite materials, etc.). For this reason the continuum with microstructure was intruduced [1]. The new model is called the micropolar continuum, and it possesses two independent kinematic quantities: the velocity vector $\vec{v}$ (macromotion) and the spin, or microrotation vector $\vec{\nu}$.

Since 1965, the micropolar theory has attracted a great deal of attention.

Paper [2] considers the stationary motion of a suspension between two coaxial cylinders. The inner cylinder with the radius $R_{1}$ is immobile, while the outer one with the radius $R_{2}\left(R_{2}>R_{1}\right)$ rotates with angular velocity $\Omega$. The axis of rotation is horizontal.

Applying the basic relations and balance laws of micropolar theory, the following result was obtained: The behaviour of the micropolar suspension between the two coaxial cylinders is described by the system of the following two coupled differential equations [2]:

$$
(\mu+k)\left[r^{2} v^{\prime \prime}+r v^{\prime}-v\right]-k r^{2} \nu^{\prime}=0
$$




$$
\gamma\left[r \nu^{\prime \prime}+\nu^{\prime}\right]+k(v r)^{\prime}-2 k r \nu=0, \quad R_{1} \leq r \leq R_{2} .
$$

Hence, $v(r)$ represents the velocity of the suspension (macromotion); $\nu(r)$ is the microrotational velocity $r$ is one of the coordinates of the cylindrical system; $\gamma, \mu$, and $k$ denote viscosity coefficients of the micropolar continuum (they are positive constants).

We treat this kind of motion of suspension when the suspension flows down the walls of the cylinders, so that the boundary conditions for the velocity $v(r)$ and the microrotation velocity $\nu(r)$ are

$$
v\left(R_{1}\right)=0, \quad v\left(R_{2}\right)=\Omega R_{2} \quad \text { and } \quad \nu\left(R_{1}\right)=0, \quad \nu\left(R_{2}\right)=0 .
$$

In this paper, the general solution $v(r)$ and $\nu(r)$ of the differential equations (1) and (2) are determined. The arbitrary constants of this solution are calculated on the basis of the boundary conditions (3). Finally, we show, that in a special case, we obtain from our result the same velocity $v(r)$ which we would have got if we solved this technical problem taking into account the laws of classical physics.

\section{Solution of the coupled system}

After multiplying (2) by $r$, the terms in the brackets of (1) and (2) are brought to the form $r^{n} y^{(n)}, y=y(r)$. These expressions may be reduced to derivatives with constant coefficients if we substitute

$$
r=R_{2} e^{-t}, \quad \text { resp. } \quad t=\ln R_{2}-\ln r .
$$

If the change of variable (4) is applied to $v(r)$ and $\nu(r)$, we determine the resulting functions

$$
V(t)=v\left(R_{2} e^{-t}\right)=v(r) \quad \text { and } \quad U(t)=\nu\left(R_{2} e^{-t}\right)=\nu(r) .
$$

Substituting (4), (5), $r v^{\prime}=-\dot{V}, r^{2} v^{\prime \prime}=\ddot{V}+\dot{V}$ and the analogous $\nu$ derivatives into equations (1) and (2) then multiplying by $r$, we obtain

$$
\begin{gathered}
\ddot{V}-V=-k R_{2}(\mu+k)^{-1} e^{-t} \dot{U}, \\
\ddot{U}-2 k \gamma^{-1} R_{2}^{2} e^{-2 t} U=k \gamma^{-1} R_{2} e^{-t}(\dot{V}-V) .
\end{gathered}
$$

After multyplying the above equation by $\gamma e^{t} /\left(k R_{2}\right)$ and differentiatiing it, we have

$$
\dot{V}-V=\frac{\gamma}{k R_{2}} e^{t} \ddot{U}-2 R_{2} e^{-t} U, \quad \ddot{V}-\dot{V}=\frac{\gamma}{k R_{2}} \frac{d\left(e^{t} \ddot{U}\right)}{d t}-2 R_{2} \frac{d\left(e^{-t} U\right)}{d t} .
$$

These two equations and equation (6) are a system of three independent differential equations. Consequently, $V, \dot{V}$ and $\ddot{V}$ can be eliminated. In this way we obtain

$$
\dddot{U}+2 \ddot{U}-M^{2} e^{-2 t} \dot{U}=0, \quad M=\left[\frac{k}{\gamma}\left(\frac{2 \mu+k}{\mu+k}\right)\right]^{1 / 2} R_{2} .
$$


Suppose the Laplace transform of $U(t)$ is denoted by $\Upsilon(s)$. Then we take the Laplace transform of both sides of equation (9). Dividing the transformed equation by $s^{2}(s+$ 2 ), then using the method of partial fraction and taking into account the boundary conditions $U(0)=\nu\left(R_{2}\right)=0$, we get

$$
\Upsilon(s)-\frac{M^{2}}{s^{2}} \Upsilon(s+2)=\frac{1}{s^{2}} \dot{U}(0)+\left(-\frac{1}{4 s}+\frac{1}{2 s^{2}}+\frac{1}{4(s+2)}\right) \ddot{U}(0) .
$$

Applying to this relation the inverse Laplace transform on this relation, we obtain the integral equation

$$
U(t)-M^{2} \int_{0}^{t} \int_{0}^{t_{1}} e^{-2 t_{2}} U\left(t_{2}\right) d t_{2} d t_{1}=t \dot{U}(0)+\left[-1+2 t+e^{-2 t}\right] \frac{\ddot{U}(0)}{4} .
$$

Introducing the new variables

$$
t_{1}=\ln \left(M / x_{1}\right), \quad t_{2}=\ln \left(M / x_{2}\right), \quad t=\ln (M / x)
$$

and setting

$$
w(\xi)=U[\ln (M / \xi)]
$$

we bring the integral equation to the form

$$
w(x)-\int_{M}^{x} \int_{M}^{x_{1}} x_{2} w\left(x_{2}\right) d x_{2} \frac{d x_{1}}{x_{1}}=\ln \left(\frac{M}{x}\right) \dot{U}(0)+\left[-1+2 \ln \frac{M}{x}+\frac{x^{2}}{M^{2}}\right] \frac{\ddot{U}(0)}{4} .
$$

If we apply to the transformed integral equation the differential operator $d / d x(x d / d x)$, we obtain the modified inhomogeneous Bessel equation with zero order

$$
x w^{\prime \prime}+w^{\prime}-x w=x M^{-2} \ddot{U}(0), \quad w=w(x) .
$$

The general solution of this differential equation is defined by

$$
w(x)=C_{1} I_{0}(x)+C_{2} K_{0}(x)-M^{-2} \ddot{U}(0) .
$$

$I_{n}$ (resp. $K_{n}$ ) is called the modified Bessel function of the first (resp., second kind) of order $n . K_{n}$ is also known as MacDonald function.

Substituting $x=M e^{-t}$ (see (10)) and (11) into the first equation (8), we get

$$
x \tilde{V}^{\prime}(x)+\tilde{V}(x)=\frac{k R_{2}}{\mu+k} \frac{x}{M}\left(C_{1} I_{0}(x)+C_{2} K_{0}(x)\right)-2 R_{2} \frac{x}{M^{3}} \ddot{U}(0) .
$$

By variation of constants we obtain a particular solution of the form $\tilde{V}_{p}=C(x) x^{-1}$. The general solution of equation (7) (resp. (12)) is now explicitely expressed:

$$
\tilde{V}=-\frac{R_{2}}{M^{3}} \ddot{U}(0) x+C_{3} \frac{1}{x}+\frac{k R_{2}}{\mu+k} \frac{1}{M}\left(C_{1} I_{1}(x)-C_{2} K_{1}(x)\right) .
$$


Substituting $e^{-t}=x / M$ into (4), using (5), $x=M e^{-t}$, (11) and (13), we find

$$
\begin{gathered}
v=-\frac{\ddot{U}(0)}{M^{2}} r+\tilde{C}_{3} \frac{1}{r}+\frac{k}{\mu+k} \frac{1}{N}\left(C_{1} I_{1}(N r)-C_{2} K_{1}(N r)\right), \\
\nu=C_{1} I_{0}(N r)+C_{2} K_{0}(N r)-\frac{\ddot{U}(0)}{M^{2}}, \quad N=M / R_{2} .
\end{gathered}
$$

These two functions satisfy equations (1) and (2) identically. To determine the arbitrary constants, we apply the boundary conditions (3) to (14) and (15). This yields

$$
\begin{gathered}
C_{1}=\frac{\Omega R_{2}^{2}}{H}\left[K_{0}\left(a_{2}\right)-K_{0}\left(a_{1}\right)\right], \quad C_{2}=\frac{\Omega R_{2}^{2}}{H}\left[I_{0}\left(a_{2}\right)-I_{0}\left(a_{1}\right)\right], \\
\frac{\ddot{U}(0)}{M^{2}}=\frac{\Omega R_{2}^{2}}{H}\left[I_{0}\left(a_{1}\right) K_{0}\left(a_{2}\right)-I_{0}\left(a_{2}\right) K_{0}\left(a_{1}\right)\right], \quad a_{j}=N R_{j}, \quad j=1,2, \\
\tilde{C}_{3}=\frac{\Omega R_{1} R_{2}^{2}}{H}\left(I_{1}\left(a_{1}\right)\left[K_{0}\left(a_{1}\right)-K_{0}\left(a_{2}\right)\right]+K_{1}\left(a_{1}\right)\left[I_{0}\left(a_{1}\right)-I_{0}\left(a_{2}\right)\right]+\right. \\
\left.+R_{1}\left[I_{0}\left(a_{1}\right) K_{0}\left(a_{2}\right)-I_{0}\left(a_{2}\right) K_{0}\left(a_{1}\right)\right]\right), \\
H=\left(R_{1}^{2}-R_{2}^{2}\right)\left[I_{0}\left(a_{1}\right) K_{0}\left(a_{2}\right)-I_{0}\left(a_{2}\right) K_{0}\left(a_{1}\right)\right]+\left[K_{0}\left(a_{1}\right)-K_{0}\left(a_{2}\right)\right] * \\
*\left[R_{1} I_{1}\left(a_{1}\right)-R_{2} I_{1}\left(a_{2}\right)\right]+\left[I_{0}\left(a_{1}\right)-I_{0}\left(a_{2}\right)\right]\left[R_{1} K_{1}\left(a_{1}\right)-R_{2} K_{1}\left(a_{2}\right)\right] .
\end{gathered}
$$

\section{Conclusion}

The classic case of suspension motion is obtained if the material constant $k$ of the micropolar suspension is equal to zero. In that case, the relation for the velocity of suspension motion is reduced to the well-known form

$$
v=\frac{\Omega}{R_{2}^{2}-R_{1}^{2}}\left[R_{2}^{2} r-\frac{R_{1}^{2} R_{2}^{2}}{r}\right] ; \quad \nu=0 .
$$

\section{REFERENCES}

[1] Eringen, A.C. and Suhubi, E.S.: Nonlinear theory of simple microelastic solids, Int. J. Engng. Sci., 2 (1964), 189-202.

[2] Cvethović, P., Tomantschger, K.W., Kuzmanović, D. and Golubović, Z.: The motion of a suspension between two coaxial cylinders, Facta Universitatis, in press.

[3] Eringen, A.C. : Simple microfluids, Int. J. Engng. Sci., 2, (1964), 205-217.

[4] Tomantschger, K.W., Cvetković, P., Kuzmanović, D. and Golubović, Z.: Solving the problem of motion of a micropolar suspension, ZAMM, in press. 